\title{
A Tutorial on Joint Radar and Communication Transmission for Vehicular Networks - Part III: Predictive Beamforming without State Models
}

\author{
Fan Liu, Member, IEEE, and Christos Masouros, Senior Member, IEEE
}

(Invited Paper)

\begin{abstract}
In Part I and II of this three-part tutorial on dualfunctional radar-communication (DFRC) design for vehicular networks, we overviewed the basics of radar and communication systems and the state of the art in DFRC respectively. As Part III of the tutorial, we address the issue of predictive beamforming for the vehicle-to-infrastructure (V2I) links without the need for explicit state evolution models. The beam tracking is done with the aid of the dual-functional radar-communication signals transmitted by the road side unit (RSU). The vehicle's location parameters are estimated by exploiting the reflected echoes signals. Given these estimates, we propose a prediction method to predict the next position of the vehicle, without specifying a state model. As a step further, we present a novel power allocation scheme for an RSU serving multiple vehicles, aiming to optimize the estimation performance while guaranteeing the downlink communication sum-rates. Finally, we verify the superiority of the proposed approaches via numerical simulations.
\end{abstract}

Index Terms - V2X, dual-functional radar-communication, beam tracking, power allocation.

\section{INTRODUCTION}

D FRC systems have recently gained considerable attention, as a spectrum-, power-, hardware-, and cost-efficient solution for combining communication and radar functionalities. Vehicular networks present a particularly suitable application of the above technology, due to the dependence of connected vehicles on high-rate communications, together with accurate anti-collision sensing and localization. Part II of this tutorial overviewed the state of the art in the area and highlighted the particular challenges in predictive beamforming for mmWave vehicular links. Conventionally, mmWave beam tracking methods are built upon communication-only feedback based protocols [1], [2]. In these schemes, pilot symbols are sent to the receiver, based on which the receiver estimates the angle of arrival (AoA) and feeds it back to the transmitter. The transmitter then formulates a transmit beam towards the angle of interest. Nevertheless, the feedback based schemes may suffer from large overhead incurred by the pilot symbols as well as the feedback procedure, which limits its usage in the vehicular network that requires low-latency transmission.

To tackle the above problems, a radar-assisted predictive beamforming design has been proposed by [3], [4], which requires the RSU to transmit DFRC signals to localize and communicate with the vehicles simultaneously. The angle and the distance of the vehicle are estimated by exploiting the echoes reflected by the vehicle's body, which replace the feedback loop in the communication-only approaches. To adapt to the high-dynamic vehicular channel, the work in [3] predicts the state of the vehicle and steer the beam towards the intended direction in advance, which relies on a state transition model. However, the state model in [3] is developed on a straight roadway geometry, which results in limited application scenarios.

In this letter, we extend the design in [3] by developing a novel predictive beamforming method, which predicts the vehicle's position based on its historical trajectory points, such that a state model is no longer needed. As a result, the proposed technique can be applied to more generic scenarios with arbitrary vehicle trajectories rather than being restricted to a straight road only. Furthermore, we propose a power allocation (PA) scheme to optimize the radar sensing performance while guaranteeing the downlink sum-rates for multiple vehicles. Finally, we validate the performance of the proposed approaches by numerical simulations.

\section{System Model}

Extending from the system model of Part II of this tutorial, we consider a mmWave mMIMO RSU, which is equipped with $N_{t}$ transmit antennas and $N_{r}$ receive antennas. The RSU is detecting $K$ vehicles, which are also single-antenna communication receivers. We denote $\theta_{k, n}$ and $d_{k, n}$ the angle and the distance of the $k$ th target at the $n$th epoch, respectively.

\section{A. Radar Sensing Model}

We assume that the state of each vehicle remains unchanged during a single transmission epoch with a duration of $\Delta T$. By denoting the transmitted DFRC signal at the $n$th transmission block and time $t$ as $\mathbf{s}_{n}(t)=\left[s_{1, n}(t), \ldots, s_{K, n}(t)\right]^{T} \in \mathbb{C}^{K \times 1}$, the received target echo signal can be expressed as

$$
\begin{aligned}
\mathbf{y}_{n}^{R}(t) & =\sum_{k=1}^{K} \kappa_{R} \beta_{k, n} \sqrt{p_{k, n}} \mathbf{b}\left(\theta_{k, n}\right) \mathbf{a}^{H}\left(\theta_{k, n}\right) \mathbf{F}_{n} \mathbf{s}_{n}\left(t-\tau_{k, n}\right) \\
& +\mathbf{z}_{n}^{R}(t),
\end{aligned}
$$

where $\kappa_{R}=\sqrt{N_{t} N_{r}}$ denotes the array gain, $\beta_{k, n}$ is the reflection coefficient, $p_{k, n}$ is the transmit power at the $k$ th beam, $\tau_{k, n}=\frac{2 d_{k, n}}{c}$ stands for the round-trip delay, $\mathbf{a}(\theta)$ and 
$\mathbf{b}(\theta)$ represent the transmit and the receive steering vectors, respectively, which are expressed as

$$
\begin{aligned}
& \mathbf{a}(\theta)=\sqrt{\frac{1}{N_{t}}}\left[1, e^{-j \pi \cos \theta}, \ldots, e^{-j \pi\left(N_{t}-1\right) \cos \theta}\right]^{T}, \\
& \mathbf{b}(\theta)=\sqrt{\frac{1}{N_{r}}}\left[1, e^{-j \pi \cos \theta}, \ldots, e^{-j \pi\left(N_{r}-1\right) \cos \theta}\right]^{T},
\end{aligned}
$$

where we assume that both arrays are uniform linear arrays (ULAs) with half-wavelength spacing between adjacent antennas. Furthermore, $\mathbf{F}_{n}=\left[\mathbf{f}_{1, n}, \mathbf{f}_{2, n}, \ldots, \mathbf{f}_{K, n}\right] \in \mathbb{C}^{N_{t} \times K}$ is the transmit beamforming matrix, with the $k$ th beamforming vector being given as

$$
\mathbf{f}_{k, n}=\mathbf{a}\left(\hat{\theta}_{k, n \mid n-1}\right),
$$

where $\hat{\theta}_{k, n \mid n-1}$ represents the $n$th angle predicted at the $(n-1)$ th epoch. The reflection coefficient $\beta_{k, n}$ is expressed as

$$
\beta_{k, n}=\varepsilon_{k, n} /\left(2 d_{k, n}\right),
$$

where $\varepsilon_{k, n}$ denotes the radar cross-section (RCS) of the vehicle. Finally, $\mathbf{z}_{n}^{R}(t) \in \mathbb{C}^{N_{r} \times 1}$ denotes the additive white Gaussian noise (AWGN) with the variance of $\sigma_{R}^{2}$.

Given the asymptotic orthogonality of the mMIMO array, the inter-beam interference vanishes and the RSU can thus process each target individually. Accordingly, the received echo of the $k$ th target is

$$
\begin{aligned}
\mathbf{y}_{k, n}^{R}(t) & =\kappa_{R} \beta_{k, n} \sqrt{p_{k, n}} \mathbf{b}\left(\theta_{k, n}\right) \mathbf{a}^{H}\left(\theta_{k, n}\right) \mathbf{f}_{k, n} s_{k, n}\left(t-\tau_{k, n}\right) \\
& +\mathbf{z}_{k, n}^{R}(t),
\end{aligned}
$$

where $\mathbf{z}_{k, n}^{R}(t)$ is the AWGN.

Following the standard radar signal processing procedure, each echo signal is matched-filtered by a time-shifted counterpart of $s_{k, n}(t)$ to obtain an estimated time-delay $\hat{\tau}_{k, n}$, which can be given by

$$
\hat{\tau}_{k, n}=2 d_{k, n} / c+z_{\tau},
$$

where $z_{\tau}$ is the estimation noise with the variance of $\sigma_{\tau}^{2}$. As such, the output of the matched filter can be expressed as

$$
\tilde{\mathbf{y}}_{k, n}^{R}=\kappa_{R} \beta_{k, n} \sqrt{p_{k, n}} \mathbf{b}\left(\theta_{k, n}\right) \mathbf{a}^{H}\left(\theta_{k, n}\right) \mathbf{a}\left(\hat{\theta}_{k, n \mid n-1}\right)+\mathbf{z}_{\theta},
$$

where $\mathbf{z}_{\theta}$ represents the output noise of the matched filter, with a variance of $\sigma_{\theta}^{2}$.

It is worth noting that the above variances are inversely proportional to the output SNR of the matched filter, which can be therefore modeled as

$$
\sigma_{\tau}^{2}=a_{1}^{2} \sigma_{R}^{2} /\left(G p_{k, n} \kappa_{R}^{2}\left|\beta_{k, n}\right|^{2} \delta_{k, n}^{2}\right), \sigma_{\theta}^{2}=a_{2}^{2} \sigma_{R}^{2} /\left(G p_{k, n}\right),
$$

where $G$ is the matched-filtering gain that equals to the energy of $s_{k, n}(t)$, and $\delta_{k, n}=\mathbf{a}^{H}\left(\theta_{k, n}\right) \mathbf{a}\left(\hat{\theta}_{k, n \mid n-1}\right)$ represents the beamforming gain. If $\hat{\theta}_{k, n \mid n-1}=\theta_{k, n}$, i.e., the angle is perfectly predicted, we have $\delta_{k, n}=1$. Otherwise, $\left|\delta_{k, n}\right|<1$. Finally, $a_{1}$ and $a_{2}$ are two scaling factors that are determined by the specific signal parameters, such as the spectrum shape and the bandwidth, etc. Note that $\sigma_{\theta}^{2}$ contains no $\beta_{k, n}, p_{k, n}$ and $\delta_{k, n}$, since they are already included in (7).
Remark 1: We remark here that the velocity parameters of the vehicles are not included in the above measurements, since the Doppler phase shift accumulated within a single transmission duration (e.g., $1 \sim 10 \mathrm{~ms}$ ) is typically not significant enough to be detected. To cope with this issue, we resort to the estimates of the distance and the angle of each vehicle to implicitly infer the velocities.

\section{B. Communication Model}

Based on the above discussion, the received communication signal at the $k$ th vehicle is given as

$$
y_{k, n}^{C}(t)=\alpha_{k, n} \kappa_{C} \sqrt{p_{k, n}} \mathbf{a}^{H}\left(\theta_{k, n}\right) \mathbf{f}_{k, n} s_{k, n}(t)+z_{k, n}^{C}(t),
$$

where $\kappa_{C}=\sqrt{N_{t}}$ is the array gain, $z_{k, n}^{C}(t)$ is the AWGN with a variance of $\sigma_{C}^{2}$, and $\alpha_{k, n}$ is the path-loss, which is given as

$$
\alpha_{k, n}=\alpha_{\mathrm{ref}} d_{k, n}^{-1} e^{j \frac{2 \pi f_{c}}{c} d_{k, n}},
$$

where $f_{c}$ denotes the carrier frequency, $\alpha_{\text {ref }}$ is a known reference path-loss measured at the distance of $1 \mathrm{~m}$. It can be seen that $\alpha_{k, n}$ can be readily estimated by estimating $d_{k, n}$.

The receive SNR at the $k$ th vehicle is therefore given as

$$
\gamma_{k, n}=p_{k, n} \kappa_{C}^{2}\left|\alpha_{k, n}\right|^{2}\left|\delta_{k, n}\right|^{2} / \sigma_{C}^{2} \triangleq p_{k, n} h_{k, n},
$$

where $\delta_{k, n}$ is the beamforming gain defined above. The achievable sum-rate of the vehicles can be thus obtained as

$$
R_{n}=\sum_{k=1}^{K} \log \left(1+\gamma_{k, n}\right)=\sum_{k=1}^{K} \log \left(1+p_{k, n} h_{k, n}\right) .
$$

Remark 2: Given the space limit, we will not elaborate on the predictive beamforming protocol for the V2I scenario considered. We refer the readers to [3] and Part II of this tutorial for more details.

\section{Parameter Estimation And Prediction}

In this section, we focus on localizing the position of the vehicle, based on which the RSU can predict its next position, and thus formulates a beam towards the direction of interest, so that a high-quality V2I link can be maintained. Without loss of generality, we consider a single vehicle and omit the index $k$ in all the notations. As a result, the variables to be estimated at the $n$th epoch can be denoted as $\mathbf{w}_{n}=\left[\theta_{n}, d_{n}, \beta_{n}\right]^{T}$.

\section{A. Parameter Estimation and Vehicle Localization}

By using the above notations, the measurement obtained at the RSU is defined as

$$
\begin{aligned}
& \mathbf{r}_{n}=\mathbf{g}\left(\mathbf{w}_{n}\right)+\mathbf{z}_{n} \\
& \triangleq\left[\begin{array}{l}
\kappa_{R} \beta_{n} \sqrt{p_{n}} \mathbf{b}\left(\theta_{n}\right) \mathbf{a}^{H}\left(\theta_{n}\right) \mathbf{a}\left(\hat{\theta}_{n \mid n-1}\right) \\
2 d_{n} / c
\end{array}\right]+\left[\begin{array}{l}
\mathbf{z}_{\theta} \\
z_{\tau}
\end{array}\right],
\end{aligned}
$$

where we have $\mathbf{z}_{n} \sim \mathcal{C N}\left(\mathbf{0}, \mathbf{Q}_{n}\right)$, with the covariance matrix $\mathbf{Q}_{n}$ being defined as $\mathbf{Q}_{n}=\operatorname{diag}\left(\sigma_{\theta}^{2} \mathbf{1}_{N_{r}}^{T}, \sigma_{\tau}^{2}\right)$.

Our goal is to estimate $\mathbf{w}_{n}$ based on $\mathbf{r}_{n}$, which is challenging due to the inherent nonlinearity in the measurement 
function $\mathbf{g}\left(\mathbf{w}_{n}\right)$. We therefore consider the following firstorder Taylor expansion at the prediction $\hat{\mathbf{w}}_{n \mid n-1}$ to approximate $\mathbf{g}\left(\mathbf{w}_{n}\right)$, which is

$$
\begin{aligned}
& \mathbf{g}\left(\mathbf{w}_{n}\right) \approx \mathbf{g}\left(\hat{\mathbf{w}}_{n \mid n-1}\right)+\left.\frac{\partial \mathbf{g}}{\partial \mathbf{w}_{n}}\right|_{\mathbf{w}_{n}=\hat{\mathbf{w}}_{n \mid n-1}}\left(\mathbf{w}_{n}-\hat{\mathbf{w}}_{n \mid n-1}\right) \\
& \triangleq \mathbf{g}\left(\hat{\mathbf{w}}_{n \mid n-1}\right)+\mathbf{G}_{n}\left(\mathbf{w}_{n}-\hat{\mathbf{w}}_{n \mid n-1}\right),
\end{aligned}
$$

where $\mathbf{G}_{n}=\left.\frac{\partial \mathbf{g}}{\partial \mathbf{w}_{n}}\right|_{\mathbf{w}_{n}=\hat{\mathbf{w}}_{n \mid n-1}}$ denotes the Jacobian matrix. Accordingly, the measurement $\mathbf{r}_{n}$ can be rewritten as

$$
\begin{aligned}
& \mathbf{r}_{n} \approx \mathbf{g}\left(\hat{\mathbf{w}}_{n \mid n-1}\right)+\mathbf{G}_{n}\left(\mathbf{w}_{n}-\hat{\mathbf{w}}_{n \mid n-1}\right)+\mathbf{z}_{n} \\
& =\mathbf{g}\left(\hat{\mathbf{w}}_{n \mid n-1}\right)+\mathbf{G}_{n} \mathbf{w}_{n}-\mathbf{G}_{n} \hat{\mathbf{w}}_{n \mid n-1}+\mathbf{z}_{n},
\end{aligned}
$$

which is linear in $\mathbf{w}_{n}$. Let us denote $\tilde{\mathbf{r}}_{n}=\mathbf{r}_{n}-\mathbf{g}\left(\hat{\mathbf{w}}_{n \mid n-1}\right)+$ $\mathbf{G}_{n} \hat{\mathbf{w}}_{n \mid n-1}$, then we have

$$
\tilde{\mathbf{r}}_{n}=\mathbf{G}_{n} \mathbf{w}_{n}+\mathbf{z}_{n} .
$$

The maximum likelihood (ML) estimator can be therefore given as

$$
\begin{aligned}
& \hat{\mathbf{w}}_{n}=\arg \min _{\mathbf{w}_{n}}\left(\tilde{\mathbf{r}}_{n}-\mathbf{G}_{n} \mathbf{w}_{n}\right)^{H} \mathbf{Q}_{n}^{-1}\left(\tilde{\mathbf{r}}_{n}-\mathbf{G}_{n} \mathbf{w}_{n}\right) \\
& =\left(\mathbf{G}_{n}^{H} \mathbf{Q}_{n}^{-1} \mathbf{G}_{n}\right)^{-1} \mathbf{G}_{n}^{H} \mathbf{Q}_{n}^{-1} \tilde{\mathbf{r}}_{n} .
\end{aligned}
$$

With the estimates $\hat{\mathbf{w}}_{n}=\left[\hat{\theta}_{n}, \hat{d}_{n}, \hat{\beta}_{n}\right]^{T}$ at hand, and by setting the RSU as the origin of the coordinate system, the Cartesian coordinates of the vehicle can be estimated as

$$
\hat{x}_{n}=\hat{d}_{n} \cos \hat{\theta}_{n}, \hat{y}_{n}=\hat{d}_{n} \sin \hat{\theta}_{n} .
$$

\section{B. Parameter Prediction}

To adapt to the high-dynamic vehicular channel, the RSU needs to predict the beamforming direction at the $(n+1)$ th epoch based on the $n$th and the previous estimates. Conventionally, this can be done by leveraging a state transition model. Given the complex traffic environment, however, it would be difficult for the RSU to acquire a tractable state model. We therefore resort to a relatively simple approach by exploiting the historical coordinates and the kinetics of the vehicle. Let us take the vehicle's movement along the $\mathrm{x}$-axis as an example, since the $y$-axis counterpart can be similarly analyzed. Based on the vehicle's kinematic equation, we have

$$
\left\{\begin{array}{l}
x_{n+1}-x_{n}=v_{x, n} \Delta T+a_{x, n} \Delta T^{2} / 2, \\
x_{n}-x_{n-1}=v_{x, n-1} \Delta T+a_{x, n-1} \Delta T^{2} / 2, \\
x_{n-1}-x_{n-2}=v_{x, n-2} \Delta T+a_{x, n-2} \Delta T^{2} / 2, \\
v_{x, n}=v_{x, n-1}+a_{x, n-1} \Delta T, \\
v_{x, n-1}=v_{x, n-2}+a_{x, n-2} \Delta T,
\end{array}\right.
$$

where $v_{x, n}$ and $a_{x, n}$ denote the velocity and the acceleration along the $\mathrm{x}$-axis at the $n$th time-slot.

By assuming that the acceleration of the vehicle does not change too much from the $(n-2)$ th to the $n$th time slots, i.e, $a_{x, n} \approx a_{x, n-1} \approx a_{x, n-2}$, one can predict $x_{n+1}$ via solving the equations above, which is obtained in the form

$$
\hat{x}_{n+1 \mid n}=3 x_{n}-3 x_{n-1}+x_{n-2} \approx 3 \hat{x}_{n}-3 \hat{x}_{n-1}+\hat{x}_{n-2} .
$$

Similarly, for the y-axis component we have

$$
\hat{y}_{n+1 \mid n}=3 y_{n}-3 y_{n-1}+y_{n-2} \approx 3 \hat{y}_{n}-3 \hat{y}_{n-1}+\hat{y}_{n-2} \text {. }
$$

It then follows that

$$
\hat{d}_{n+1 \mid n}=\sqrt{\hat{x}_{n+1 \mid n}^{2}+\hat{y}_{n+1 \mid n}^{2}}, \hat{\theta}_{n+1 \mid n}=\arctan \frac{\hat{y}_{n+1 \mid n}}{\hat{x}_{n+1 \mid n}} .
$$

To predict the reflection coefficient $\beta_{n+1}$, note that

$$
\beta_{n}=\varepsilon_{n} /\left(2 d_{n}\right) \rightarrow \varepsilon_{n}=2 \beta_{n} d_{n} .
$$

Since the RCS may not change too much during a small number of transmission slots, $\varepsilon_{n+1}$ can be predicted by averaging over the previous $n$th and $(n-1)$ th measurements, which is

$$
\hat{\varepsilon}_{n+1 \mid n} \approx\left(2 \beta_{n} d_{n}+2 \beta_{n-1} d_{n-1}\right) / 2 \approx \hat{\beta}_{n} \hat{d}_{n}+\hat{\beta}_{n-1} \hat{d}_{n-1},
$$

which leads to

$$
\hat{\beta}_{n+1 \mid n}=\hat{\varepsilon}_{n+1 \mid n} /\left(2 \hat{d}_{n+1 \mid n}\right) .
$$

Hence we finally arrive at

$$
\hat{\mathbf{w}}_{n+1 \mid n}=\left[\hat{\theta}_{n+1 \mid n}, \hat{d}_{n+1 \mid n}, \hat{\beta}_{n+1 \mid n}\right]^{T} .
$$

\section{POWER Allocation}

In this section, we discuss the PA problem among multiple vehicles, which aims to optimize the radar sensing performance while guaranteeing the communication qualityof-service (QoS). In particular, we are interested in minimizing the summation of the Carmér-Rao Bound (CRB) of $K$ vehicles, subject to the communication sum-rate constraints. As the transmit power is re-allocated for every transmission epoch, we will focus on a single epoch with multiple vehicles, and omit the index $n$ in the following discussion for notational convenience, i.e., $\mathbf{w}_{k}=\left[\theta_{k}, d_{k}, \beta_{k}\right]^{T}$.

\section{A. Carmér-Rao Bound}

Following the similar notations as in Sec. II, the measurement model for the $k$ th vehicle at the RSU can be formulated as

$$
\mathbf{r}_{k}=\mathbf{g}\left(\mathbf{w}_{k}\right)+\mathbf{z}_{k},
$$

where the covariance matrix of the measurement noise $\mathbf{z}_{k}$ is the same as in Sec. II, and is now denoted as $\mathbf{Q}_{k}$. The Fisher Information Matrix (FIM) can be computed as

$$
\mathbf{J}_{k}=\left(\frac{\partial \mathbf{g}}{\partial \mathbf{w}_{k}}\right)^{H} \mathbf{Q}_{k}^{-1}\left(\frac{\partial \mathbf{g}}{\partial \mathbf{w}_{k}}\right)
$$

where $\frac{\partial \mathbf{g}}{\partial \mathbf{w}_{k}}$ is the Jacobian matrix. It is known that

$$
\mathbb{E}\left(\left(\hat{\mathbf{w}}_{k}-\mathbf{w}\right)\left(\hat{\mathbf{w}}_{k}-\mathbf{w}\right)^{H}\right) \succeq \mathbf{J}_{k}^{-1} \triangleq \mathbf{C}_{k},
$$

where $\mathbf{C}_{k}$ is defined as the CRB matrix. Therefore we have

$$
\begin{aligned}
& \mathbb{E}\left(\left(\hat{\beta}_{k}-\beta_{k}\right)^{2}\right)+\mathbb{E}\left(\left(\hat{\theta}_{k}-\theta_{k}\right)^{2}\right) \\
& +\mathbb{E}\left(\left(\hat{d}_{k}-d_{k}\right)^{2}\right) \geq \operatorname{tr}\left(\mathbf{C}_{k}\right) .
\end{aligned}
$$




\section{B. Problem Formulation and Analysis}

Our aim is to minimize the summation of the CRB of all the target vehicles, subject to communication rate constraints. Let us first denote $\tilde{\mathbf{Q}}_{k}=p_{k} \mathbf{Q}_{k}$, which is a normalized covariance matrix. Then we have

$$
\begin{aligned}
& \operatorname{tr}\left(\mathbf{C}_{k}\right)=\operatorname{tr}\left(\left(p_{k}\left(\frac{\partial \mathbf{g}}{\partial \mathbf{w}_{k}}\right)^{H} \tilde{\mathbf{Q}}_{k}^{-1}\left(\frac{\partial \mathbf{g}}{\partial \mathbf{w}_{k}}\right)\right)^{-1}\right) \\
& \triangleq p_{k}^{-1} \operatorname{tr}\left(\tilde{\mathbf{C}}_{k}\right) .
\end{aligned}
$$

By denoting $c_{k}=\operatorname{tr}\left(\tilde{\mathbf{C}}_{k}\right)$, the optimization problem can be formulated as

$$
\begin{aligned}
\min _{\mathbf{p}} & \sum_{k=1}^{K} c_{k} p_{k}^{-1} \\
\text { s.t. } & \sum_{k=1}^{K} \log _{2}\left(1+h_{k} p_{k}\right) \geq R_{0}, \\
& 1^{T} \mathbf{p} \leq P_{T}, p_{k} \geq 0, \forall k,
\end{aligned}
$$

where $\mathbf{p}=\left[p_{1}, p_{2}, \ldots p_{K}\right]^{T}$ is the PA vector to be optimized, $h_{k}$ is defined in (11) by omitting the subscript $n, R_{0}$ is the required sum-rate threshold, and $P_{T}$ is the transmit power budget.

While the PA problem is convex and can be easily solved via numerical tools, we will show that a closed-form solution is obtainable under some certain conditions. Let us firstly formulate the Lagrangian multiplier as follows

$$
\begin{aligned}
& \mathcal{L}\left(\mathbf{p}, \lambda, \mu, v_{k}\right) \\
& =\sum_{k=1}^{K} c_{k} p_{k}^{-1}+\lambda\left(-\sum_{k=1}^{K} \log _{2}\left(1+h_{k} p_{k}\right)+R_{0}\right) \\
& +\mu\left(1^{T} \mathbf{p}-P_{T}\right)-\sum_{k=1}^{K} \eta_{k} p_{k}
\end{aligned}
$$

where $\lambda, \mu$ and $\eta_{k}$ are dual variables. As a step further, the Karush-Kuhn-Tucker (KKT) conditions with respect to problem (32) can be formulated as

$$
\begin{array}{r}
\frac{\partial \mathcal{L}}{\partial p_{k}}=-c_{k} p_{k}^{-2}-\frac{\lambda h_{k}}{\left(1+h_{k} p_{k}\right) \ln 2}+\mu-\eta_{k}=0, \forall k \\
\lambda\left(-\sum_{k=1}^{K} \log _{2}\left(1+h_{k} p_{k}\right)+R_{0}\right)=0, \lambda \geq 0 \\
\mu\left(1^{T} \mathbf{p}-P_{T}\right)=0, \mu \geq 0, \\
\eta_{k} p_{k}=0, \eta_{k} \geq 0, \forall k \\
1^{T} \mathbf{p} \leq P_{T}, \\
p_{k} \geq 0, \forall k
\end{array}
$$

One can immediately observe that $p_{k}>0, \forall k$. This is because if $p_{k}=0$, the objective function becomes unbounded. Based on (34d), we have $\eta_{k}=0$. Moreover, it can be noted that the power constraint should be strictly satisfied, i.e., $1^{T} \mathbf{p}=$ $P_{T}$. This can be proved by contradiction. Assume that the summation of the optimal $p_{k}$ is less than $P_{T}$. One can always increase any $p_{k}$ to reach a summation of $P_{T}$, which further
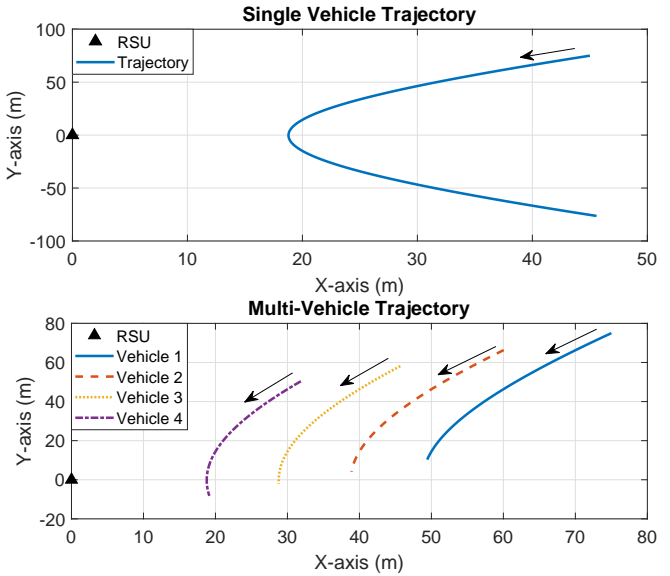

Fig. 1. Trajectories for single- and multi-vehicle scenarios.

reduces the objective function while increasing the sum-rate. Therefore, the power budget should be fully exploited. Given the complementary slackness of the KKT conditions, we have $\mu>0$.

Theorem 1. Problem (32) has closed-form solutions if

$$
R_{0} \in\left(0, R_{t h}\right] \cup R_{\max }
$$

where

$$
R_{t h}=\sum_{k=1}^{K} \log _{2}\left(1+\frac{h_{k} \sqrt{c_{k}}}{\sum_{k=1}^{K} \sqrt{c_{k}}} P_{T}\right),
$$

and $R_{\max }$ is the water-filling rate given the power budget $P_{T}$ and the channel coefficients $h_{k}$.

Proof. Since $\eta_{k}=0$, we have

$$
\frac{\partial \mathcal{L}}{\partial p_{k}}=-c_{k} p_{k}^{-2}-\frac{\lambda h_{k}}{\left(1+h_{k} p_{k}\right) \ln 2}+\mu=0 .
$$

In the case $\lambda=0$, according to the complementary slackness we have

$$
\sum_{k=1}^{K} \log _{2}\left(1+h_{k} p_{k}\right)>R_{0}
$$

and

$$
\mu=c_{k} p_{k}^{-2} \text {. }
$$

From the above, it follows that $p_{k}=\sqrt{\frac{c_{k}}{\mu}}$. Hence the power constraint can be rewritten as

$$
\sum_{k=1}^{K} p_{k}=\sum_{k=1}^{K} \sqrt{\frac{c_{k}}{\mu}}=\sqrt{\frac{1}{\mu}} \sum_{k=1}^{K} \sqrt{c_{k}}=P_{T} .
$$

Therefore

$$
\mu=\left(\sum_{k=1}^{K} \sqrt{c_{k}}\right)^{2} / P_{T}^{2},
$$

which results in the closed-form solution

$$
p_{k}=\sqrt{c_{k}} P_{T} / \sum_{k=1}^{K} \sqrt{c_{k}} \text {. }
$$


It can be seen that when $R_{0} \leq R_{t h}$, the sum-rate constraint is inactive, and hence $\lambda=0$. One can readily obtain the optimal PA expression (42).

When $R_{0}=R_{\max }$, it is known that the water-filling powerallocation is unique and optimal, and the feasible region of the problem contains only a single point. Therefore, the solution to the problem is the water-filling solution, which can be again expressed in closed form. This completes our proof.

\section{Numerical Results}

In this section, we provide numerical results based on Monte Carlo simulations to verify the effectiveness of the proposed approaches. For simplicity, we set the noise variances as $\sigma_{R}^{2}=\sigma_{C}^{2}=1$, and use normalized reference channel coefficients for both radar and communication. Accordingly, we define the transmit SNR as $\frac{P_{T}}{\sigma_{R}^{2}}$. The matched filtering gain is assumed to be $G=10$. For the measurement noise variances, we set $a_{1}=2.1 \times 10^{-7}$ and $a_{2}=1$. Without loss of generality, we study two cases for single- and multi-vehicle scenarios, respectively, with the trajectories shown in Fig. 1.

We first show in Figs. 2(a)-2(b) the simulation results of tracking a single vehicle. In Fig. 2(a), the estimation performance for both angle and distance is shown in terms of the cumulative distribution function (CDF) for estimation error, with varied number of transmit and receive antennas. It can be observed that by using the proposed technique, the angle and the distance estimation errors can be reduced to the levels of $0.1^{\circ}$ and $0.5 \mathrm{~m}$, respectively. Moreover, it is important to note that larger antenna array does not necessarily lead to better performance, despite that a higher array gain is obtainable. This is because larger antenna array typically generates narrower beams, which is more likely to miss the target vehicle, especially when the vehicle is moving at a relatively high speed.

The impact of beam misalignment is explicitly shown in Fig. 2(b), where we plot the variation of the achievable communication rate for the vehicle along its trajectory. Again, with low transmit SNR, the 128-antenna RSU performs worse than its 64-antenna counterpart. Nevertheless, when the SNR is increased to $20 \mathrm{~dB}$, the misalignment probability becomes small, and the 128-antenna system achieves higher rates due to its higher array gain.

We further show the simulation results for tracking 4 vehicles in Figs. 2(c)-2(d) with $N_{t}=N_{r}=64$ and SNR $=12 \mathrm{~dB}$, where the water-filling PA is employed as a benchmark, and the rate threshold is set as $R_{0}=0.95 R_{\max }$. It can be seen in Fig. 2(c) that by relying on the proposed PA approach, the estimation performance for both angle and distance can be significantly improved compared to the water-filling PA. In Fig. 2(d), we observe that the achievable sum-rate by using the proposed approach is higher than that of the water-filling PA thanks to more accurate vehicle localization and beam tracking.

\section{CONCLUSION}

In this letter, we have addressed the issue of predictive beamforming design for the V2I links by leveraging the radar functionality equipped on the RSU. In particular, in Part II of this tutorial we have discussed SoA approaches that estimate the angle and the distance of the vehicle by exploiting the reflected echoes of the transmitted DFRC signals. As a step beyond the SoA, in this letter we have proposed a prediction method to predict vehicle's next coordinates without specifying a state transition model. Furthermore, we have proposed a novel power allocation design for multiple vehicles, aiming for optimizing the estimation performance while ensuring the sum-rate constraints. Numerical results have been provided, showing that the predictive beamforming design can achieve superior sensing and communication performance given complex vehicle trajectories, and that the proposed power allocation outperforms the conventional beam tracking and water-filling, leading to increased beamforming gain and a consequently higher sum-rate.

\section{REFERENCES}

[1] D. Zhang, A. Li, M. Shirvanimoghaddam, P. Cheng, Y. Li, and B. Vucetic, "Codebook-based training beam sequence design for millimeter-wave tracking systems," IEEE Trans. Wireless Commun., vol. 18, no. 11, pp. 5333-5349, Nov 2019.

[2] V. Va, H. Vikalo, and R. W. Heath, "Beam tracking for mobile millimeter wave communication systems," in 2016 IEEE Global Conference on Signal and Information Processing (GlobalSIP), Dec 2016, pp. 743-747.

[3] F. Liu, W. Yuan, C. Masouros, and J. Yuan. (2020) "Radar-assisted predictive beamforming for vehicular links: Communication served by sensing". Submitted to IEEE Trans. Wireless Commun. [Online]. Available: https://arxiv.org/abs/2001.09306

[4] W. Yuan, F. Liu, C. Masouros, J. Yuan, D. W. K. Ng, and N. GonzalezPrelcic. (2020) "Bayesian predictive beamforming for vehicular networks: A low overhead joint radar-communication approach". Submitted to IEEE Trans. Wireless Commun. [Online]. Available: https://arxiv.org/abs/2005.07698 